SUBJECT AREAS:

ENDOPLASMIC

RETICULUM

MOUSE

Received

7 February 2014

Accepted

29 May 2014

Published

23 June 2014

Correspondence and requests for materials should be addressed to A.S.L. (amylee@usc. edu)

* Current address:

Hamon Center for

Therapeutic Oncology

Research, Departments of Surgery and Pharmacology,

University of Texas

Southwestern Medical

Center, Dallas, TX,

USA.

\section{Differential requirement of GRP94 and GRP78 in mammary gland development}

\author{
Genyuan Zhu', Miao Wang ${ }^{1 *}$, Benjamin Spike ${ }^{2}$, Peter C. Gray ${ }^{3}$, Jieli Shen', Sung-Hyung Lee ${ }^{4}$, Si-Yi Chen $^{4}$ \\ \& Amy S. Lee'
}

'Department of Biochemistry and Molecular Biology, University of Southern California, Keck School of Medicine, USC Norris Comprehensive Cancer Center, Los Angeles, CA, USA, ${ }^{2}$ Gene Expression Laboratories, The Salk Institute for Biological Studies, La Jolla, CA, USA, ${ }^{3}$ Clayton Foundation Laboratories for Peptide Biology, The Salk Institute for Biological Studies, La Jolla, CA, USA, ${ }^{4}$ Department of Molecular Microbiology and Immunology, University of Southern California, Keck School of Medicine, Los Angeles, CA, USA.

Glucose Regulated Protein (GRP) 94 and GRP78 are critical molecular chaperones and regulators of signaling. Conditional knockout mouse models have revealed tissue specific requirements for GRP94 and GRP78, including selection for allele retention in specific cell types. Here we report the consequences of mammary-targeted knockout of these GRPs. Our studies revealed that MMTV-Cre, Grp94 $4^{f / f}$ mammary glands, despite GRP94 deficiency, exhibited normal proliferation and ductal morphogenesis. Interestingly, $M M T V$-Cre, $G r p 78^{f / f}$ mammary glands displayed only slightly reduced GRP78 protein levels, associating with the retention of the non-recombined Grp 78 floxed alleles in isolated mammary epithelial cells and displayed phenotypes comparable to wild-type glands. In contrast, transduction of isolated Grp7 $8^{f / f}$ mammary epithelial stem/progenitor cells with adenovirus expressing GFP and Cre-recombinase was successful in GRP78 ablation, and the GFP sorted cells failed to give rise to repopulated mammary glands in de-epithelialized recipient mice. These studies imply GRP78, but not GRP94, is required for mammary gland development.

M ouse mammary gland development starts during embryogenesis and a rudimentary ductal system is evident within the mammary fat pad at birth ${ }^{1,2}$. After birth, the mammary gland grows at the similar rate as other organs and remains confined to the nipple proximal end of the fat pad, until puberty when expansive growth of the epithelial mammary tree occurs ${ }^{1,2}$. In maturing mice, club-shaped terminal end buds (TEB) appear at the ductal tips that break through the fat pad and mammary mesenchyme as the ductal structures proliferate and elongate ${ }^{1,2}$. Thus, in mature virgin female mice, the ductal system extends throughout the entire fat pad with secondary branches budding from the primary ducts ${ }^{1,2}$. Following the extensive post-pubertal ductal elaboration in the mammary gland, proliferating epithelial cells generate alveolar buds under hormonal changes during pregnancy. Alveolar buds differentiate into alveoli which subsequently synthesize and secrete milk during lactation $^{1,2}$. After weaning, lack of milk demand causes an involution process where widespread apoptosis and tissue remodeling transform a lactating gland back to a simple ductal architecture ${ }^{3}$.

All stages of mammary development are intricately regulated by the extracellular matrix (ECM) environment and diverse hormones ${ }^{4-9}$. Microenvironmental adhesive signals from the ECM have a central role in all aspects of mammary development and function, such as branching morphogenesis, formation of polarized mammary ducts, and alveolar outgrowth/involution that occurs after lactation ${ }^{4}$. A mammary gland is composed of stroma and a bilayered epithelium with inner luminal cells and outer myoepithelial cells ${ }^{4}$. Basement membrane, which is a specialized form of ECM, separates epithelium and stroma ${ }^{4}$. As sensors and transducers of ECM signals, integrins expressed in epithelial cells of the mammary gland play critical roles in regulation of mammary gland development ${ }^{5,6}$. Pubertal mammary gland growth is also influenced by growth hormone (GH), estrogen and insulin-like growth factor-1 (IGF-1) $)^{7-9}$.

The function of these developmental regulators depends on a number of upstream processes that ensure their proper presentation and function. In the lumen of endoplasmic reticulum (ER), transmembrane and secretory proteins undergo a series of maturation processes, including folding into native conformation and posttranslational modification ${ }^{10}$. Protein folding quality control exists in the ER to avoid accumulation of misfolded and unfolded proteins. Important contributors to this quality control system are the glucose regulated proteins (GRPs) $)^{11,12}$. GRP94, an abundant glycoprotein in the ER also known as HSP90B1, directs the folding and assembly of a selective class of secreted and membrane proteins, such as major histocompatibility class II 
a

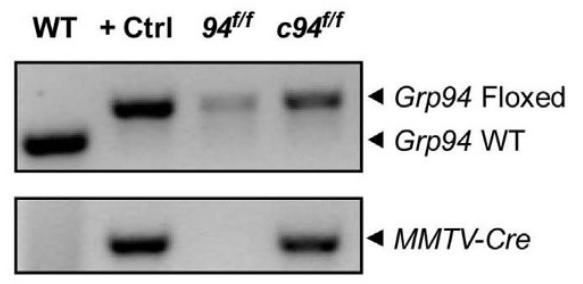

b

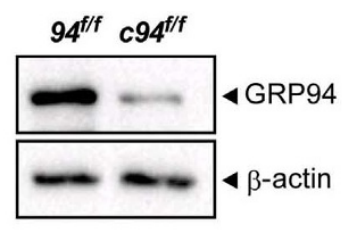

d

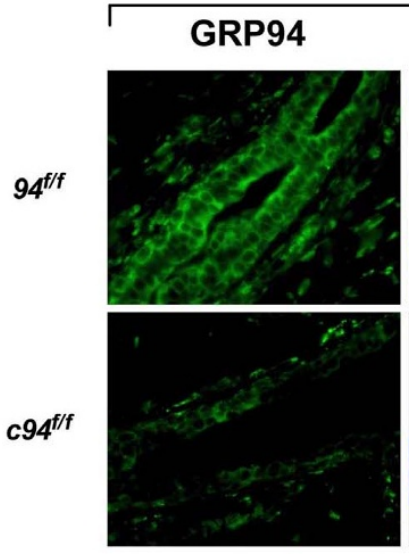

4 mos
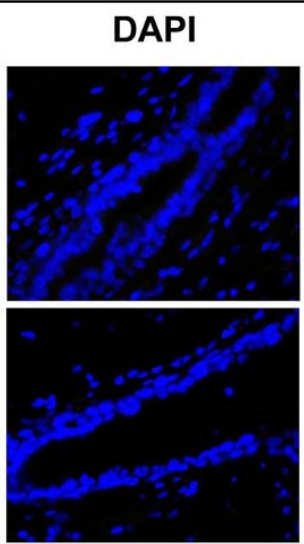
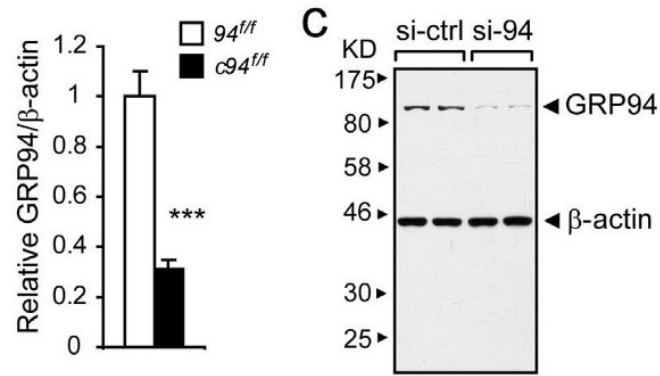

IHC

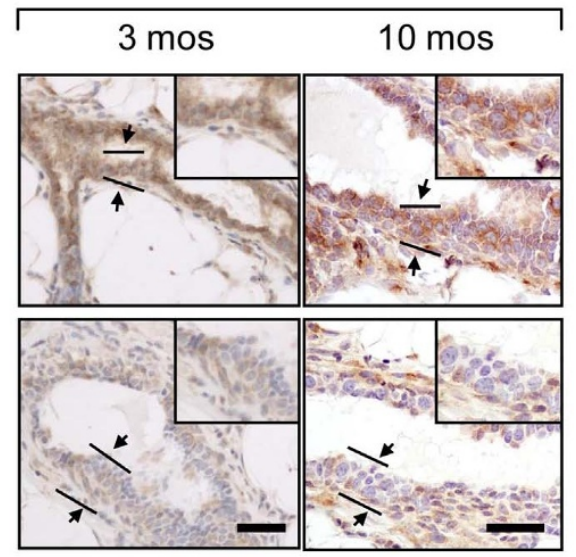

Figure 1 Generation of MMTV-Cre mediated GRP94 knockout mouse model. (a) Representative PCR genotyping results from tails of $94^{f / f}$ and $c 94^{f / f}$ mice. The alleles are indicated. Cropped gels were shown and the full-length gels were indicated in Supplementary Fig. 2a. (b) Representative Western blot detection of GRP94 levels in mammary epithelial cells isolated from $94^{f / f}$ and $c 94^{f / f}$ glands at 2.5 months, with $\beta$-actin serving as loading control. Quantitation of GRP94 level after normalization to the $\beta$-actin level is shown on right $(\mathrm{n}=2$ per genotype). Data are presented as mean \pm S.E. $\mathrm{p}<0.001$. Cropped blots were shown and the full-length blots were indicated in Supplementary Fig. 3a. (c) Western blot analysis of HBL100-HER2 cells transfected with either si-control (si-ctrl) or si-Grp94 (si-94) for detection of GRP94, with $\beta$-actin as loading control. (d) Immunofluorescent (IF) and immunohistochemical (IHC) staining of GRP94 in mammary glands from $94^{f / f}$ and $c 94^{f f}$ mice at the indicated ages. PMSG was injected two days before euthanasia of 4 and 10 month old mice to synchronize estrous cycle. Green or brown color depicts GRP94 staining. Blue color depicts DAPI staining. Scale bars show $50 \mu \mathrm{m}$ and are applicable to all sections. Negative controls for staining were shown in Supplementary Fig. $4 \mathrm{a}$, b.

(MHC II), IGF-1, multiple toll like receptors (TLRs) and a subset of integrins ${ }^{13-15}$. Some client proteins of GRP94 participate in the normal developmental process of mouse mammary gland, such as $\alpha 2$ integrin and IGF-1 ${ }^{16,17}$. GRP94 was essential for the expression of $\alpha 2$ integrin of the hematopoietic system ${ }^{16}$. Ablation of GRP94 in murine striated muscle caused smaller skeletal muscles by inhibiting local IGF-1 production ${ }^{17}$.

The most abundant ER chaperone is GRP78, also referred to as BiP and HSPA5. GRP78 is a multifunction protein not only responsible for ER protein folding and assembly but also for targeting misfolded proteins for degradation and regulating the unfolded protein response (UPR) by maintaining the ER stress transducers in inactive forms in non-stressed cells ${ }^{12,18,19}$. Recently, it was demonstrated that GRP78 can be found on the cell surface, interacting with cell surface proteins and acting as a co-receptor in signaling pathways ${ }^{20-24}$. In cell culture systems, as well as in mouse knockout models, GRP78 has been demonstrated to be essential for many cellular processes including embryogenesis ${ }^{25}$, adipogenesis ${ }^{26}$, maintenance of hematopoietic homeostasis $^{27}$, as well as protection from neuronal apoptosis ${ }^{28}$. In contrast, depletion of GRP78 in the mouse prostate epithelium through homozygous knockout of the Grp78 floxed alleles mediated by the probasin-Cre recombinase system did not affect prostate morphology, growth or function ${ }^{29}$. This raises the interesting question whether GRP78 is required for the development and function of other epithelial cell types, such as those of the mammary glands. Previous studies showed that cell surface GRP78 is a necessary mediator of Cripto signaling in mammary epithelial and embryonic stem cells $^{22}$. We recently observed that IGF-1 receptor signaling regulates GRP78 expression via the PI3K/AKT/mTORC1 axis $^{30}$, suggesting that IGF-1 may influence mammary gland development via GRP78 regulation.

To test directly whether GRP94 and GRP78 are pivotal for postnatal mammary gland development and function, we created GRP94 and GRP78 conditional knockout mouse models in the mouse mammary epithelium directed by the mouse mammary tumor virus (MMTV)-Cre recombinase system and examined their phenotypes. The MMTV-Cre recombinase of the transgenic strain (line D) used in this study shows activation by postnatal day 22 and has been commonly used to delete genes in both luminal epithelial and myoepithelial cells of mammary gland ${ }^{31,32}$. Additionally, we used adenoviral Cre-recombinase to deplete GRP78 in isolated mammary epithelial stem/progenitor cells followed by transplant assays. Our studies reveal some unexpected results and provide new evidence that the GRPs are differentially required for mammary gland development.

\section{Results}

Generation of MMTV-Cre mediated GRP94 knockout mouse model. To generate mammary epithelium-specific GRP94 knockout mice, Grp94/f $\left(94^{f / f}\right)$ mice bearing floxed $(f)$ alleles ${ }^{33}$ were crossed with the $M M T V-C r e$ transgenic mice ${ }^{31}$. The resulting MMTV-Cre, Grp $94^{f /+}\left(c 94^{f /+}\right)$ mice were bred with $94^{f / f}$ mice to generate $c 94^{f / f}$ mice, with sibling $94^{f / f}$ mice devoid of the Cre-allele serving as wild-type controls (Supplementary Figure 1a). The 
a

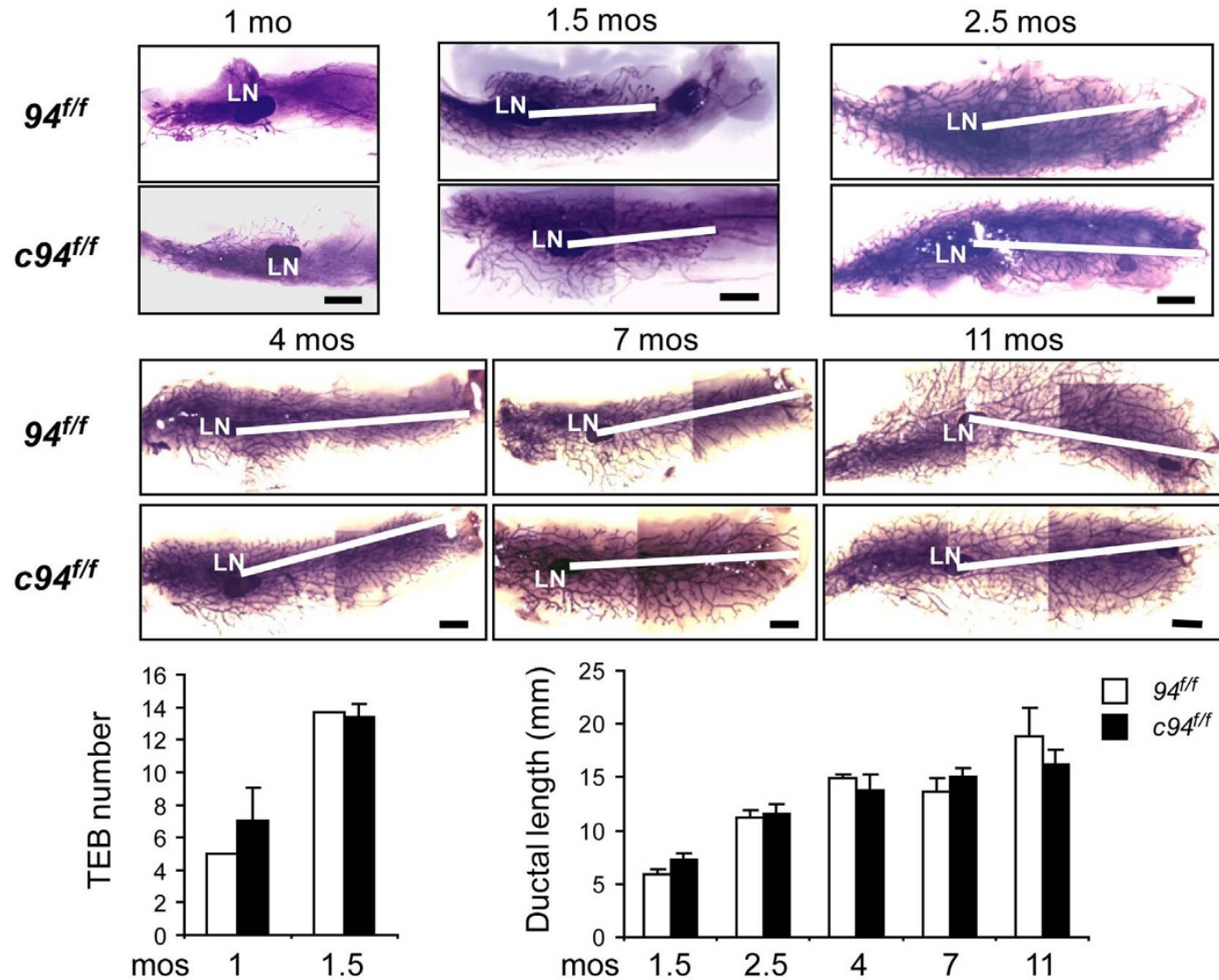

Figure $2 \mid$ Ductal morphogenesis of $c 94^{f / f}$ mammary glands. (a) Representative whole-mount staining of mammary glands from $94^{f / f}$ and $c 94^{f / f}$ mice at 1 , $1.5,2.5,4,7$ and 11 month old as indicated on top. White bars denote the length between center of the lymph node and distal end of the longest ducts in $94^{f / f}$ and $c 94^{f / f}$ glands. LN, lymph node. Scale bars show $2 \mathrm{~mm}$ and are applicable to all sections. (b) Quantitation of TEB number and ductal length of $94^{f / f}$ and $\mathrm{c} 94^{f / f}$ mammary glands at times indicated. For each genotype, $\mathrm{n}$ varies from 2 to 11 . Data are presented as mean \pm S.E. PMSG was injected two days before euthanasia of 4,7 and 11 month old mice.

genotypes of mice harboring the Grp94 floxed alleles and the MMTVCre allele were determined by PCR using genomic DNA extracted from tails of $94^{f f f}$ and $c 94^{f f f}$ mice (Figure 1a). $c 94^{f f f}$ mice were born with expected Mendelian ratio and phenotypically normal compared with their $94^{\text {fff }}$ littermates. Corresponding to knockout of GRP94, the epithelial cells isolated from the $c 94^{f f f}$ mammary glands showed $70 \%$ reduction in GRP94 protein level, compared to $94^{\text {fff }}$ glands (Figure 1b). The GRP94 protein band was also substantially reduced in human breast HBL100-HER2 cells treated with siRNA against GRP94, validating the specificity of the monoclonal antibody used for GRP94 detection (Figure 1c). Immunofluorescent (IF) staining showed efficient knockdown of GRP94 expression in the epithelial cells lining the c94/f mammary ducts (Figure 1d). Immunohistochemical (IHC) staining further confirmed the IF staining results and showed reduction of GRP94 expression persisted at least up to 10 months in the $c 94^{\text {t/f }}$ mammary glands (Figure 1d).

$c 94^{f f f}$ mammary glands displayed normal pubertal and postpubertal ductal morphogenesis. Mammary glands from $94^{\text {fff }}$ and $c 94^{f f f}$ mice at different time points were whole-mounted to examine ductal morphogenesis. Around the onset of puberty (1 month), we noticed comparable ductal outgrowth in $94^{\text {fff }}$ and $c 94^{\text {fff }}$ mammary glands (Figure 2a). There are similar numbers of TEBs at the ductal tips in $94^{f f f}$ and $c 94^{f f f}$ glands at 1 and 1.5 months (Figure 2a, b). Similar to control glands, the ducts in mutant glands completely filled the mammary fat pad by 2.5 months (Figure 2a). Following post-pubertal growth, we observed formation of tertiary branches and gradual ductal elaboration in both $94^{f f f}$ and $c 94^{f / f}$ mammary glands from 4 to 11 months (Figure 2a). Measurement of ductal distance demonstrated that $c 94^{f / f}$ mammary glands exhibited normal branching from 1.5 to 11 months (Figure 2b). Hematoxylin and eosin (H\&E) staining confirmed histoarchitecture of TEBs at 1.5 months and mammary ducts at 4,7 and 11 months were indistinguishable between the $94^{f f f}$ and $c 94^{f / f}$ mice (Figure 3a). As shown by PCNA staining, $c 94^{f f}$ mammary epithelium showed equivalent proliferation compared to WT mammary epithelium at 1.5 and 4 months (Figure 3b, c). Furthermore, the $c 94^{t f}$ mice gave birth to normal numbers of pups and fed their pups properly. H\&E staining demonstrated normal secretory alveolar structures in lactating $c 94^{f / f}$ mammary glands (Fig. 3a).

MMTV-Cre, Grp78 fff mammary glands showed only slight reduction of GRP78 expression. The breeding scheme to generate the MMTV-Cre, Grp $78^{f f f}$ mouse model ( $c 78^{f f f}$ mice) was shown in Supplementary Figure $1 \mathrm{~b}$. Using the genomic DNA extracted from tails, we detected the MMTV-Cre allele in $c 78^{f f f}$ mice, but not $78^{f f f}$ mice (Figure 4a). Western blot analysis showed around 30\% reduction of GRP78 protein level in mammary epithelial cells isolated from $c 78^{f f}$ glands, compared with $78^{f f f}$ glands (Figure $4 \mathrm{~b}$ ). To validate specificity of the anti-GRP78 antibody for subsequent staining, we showed that as expected, the antibody detected basal GRP78 expression which was elevated in A549 cells treated with the ER stress inducer tunicamycin, and in cells treated with siRNA against GRP78, both the basal and induced level of GRP78 was nearly eliminated (Figure 4c). As revealed by IF and IHC staining, GRP78 expression was detectable in the $78^{8 / f}$ mammary epithelium (Figure 4d). Corresponding to Western blot results, we only observed 

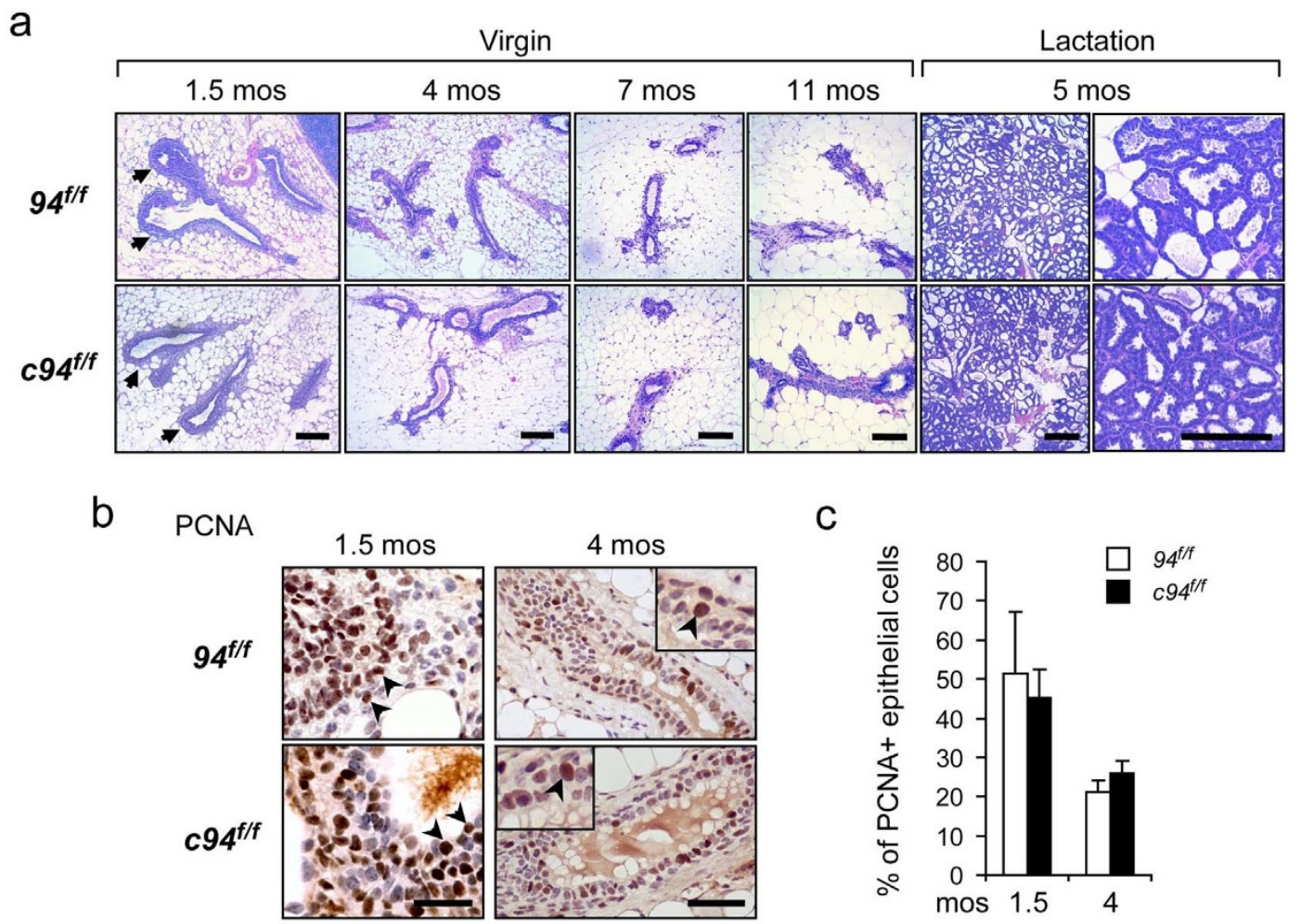

C

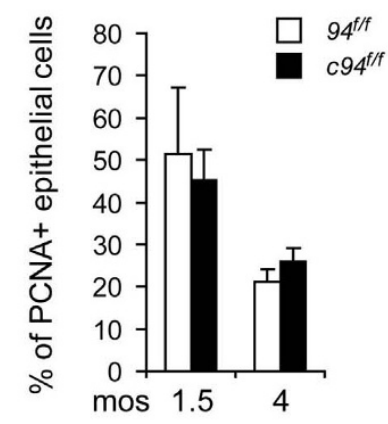

Figure $3 \mid$ Morphology and proliferation of $c 94^{f / f}$ mammary epithelium. (a) H\&E staining of $94^{f / f}$ and $c 94^{f / f}$ mammary glands from $1.5,4,7$ and 11 month old virgin mice and 5 month old lactating mice on day 7 after parturition. Arrows denote TEBs. Scale bars show $200 \mu \mathrm{m}$ and are applicable to all sections. (b) IHC staining of cell proliferative marker PCNA (brown nuclei) in mammary glands from $94^{f / f}$ and $c 94^{f / f}$ mice at 1.5 and 4 months. Scale bars show $100 \mu \mathrm{m}$ and are applicable to all sections. Arrow heads point to PCNA positive cells. (c) Quantitation of percentage of PCNA positive epithelial cells in panel b. For each genotype, $\mathrm{n}$ varies from 2 to 4 . PMSG was injected two days before euthanasia of 4,7 and 11 month old mice.

slight reduction of GRP78 expression in the $c 78^{f / f}$ mammary epithelium at various time points (1.5, 2 and 4 months) after the reported activation time span for the MMTV-Cre recombinase (Figure 4d).

$c 78^{f / f}$ mammary glands showed normal pubertal and post-pubertal ductal morphogenesis. Whole-mounted $78^{f / f}$ and $c 78^{f / f}$ mammary glands were examined for ductal morphogenesis. Examining mammary glands at the onset of puberty (1 month), maturation (2 months) or post-pubertal ages ( 4 and 7 months), we observed normal branch morphology in both $c 78^{f / f}$ and $78^{f / f}$ mammary glands (Figure 5a). Comparable numbers of TEBs were observed at the ductal tips in $78^{f / f}$ and $c 78^{f / f}$ mammary glands at 1 and 2 months (Figure 5a, b). From 2 to 7 months, $c 78^{f / f}$ mammary glands exhibited similar ductal distance as $78^{f f f}$ glands at all the time points examined (Figure 5b). H\&E staining also showed comparable histoarchitecture of TEBs at 1 month and mammary ducts at 2,4 and 5.5 months in the $78^{f / f}$ and $c 78^{f / f}$ mice (Figure 6a). IHC staining of a luminal cell marker (E-cadherin) and a myoepithelial cell marker ( $\alpha$-SMA, $\alpha$-smooth muscle actin) further demonstrated normal TEB structure in $c 78^{f / f}$ mammary glands at 1 month (Figure 6b). Similar numbers of ducts were also observed in $78^{f f f}$ and $c 78^{f f f}$ mammary glands at 2, 4 and 5.5 months (Figure 6c). Similar to the $c 94^{f / f}$ mice, $c 78^{f / f}$ female mice were fertile, gave birth to normal number of pups, and exhibited normal secretory alveolar structures in lactating $c 78^{f / f}$ mammary glands (Figure 6a).

$78^{f / f}$ and $c 78^{f / f}$ mammary glands exhibited similar distribution of different sub-lineages and outgrowth in mammary transplant. To better understand the basis for the phenotypic similarities observed between $c 78^{f / f}$ and $78^{f / f}$ glands, we isolated mammary epithelial cells from virgin $c 78^{f / f}$ and $78^{f / f}$ mice and analyzed their cellular content by flow cytometry. Representative flow cytometric analyses showed similar distribution of myoepithelial cells (MYO), mammary repopulating unit (MRU) and luminal epithelial cells enriched fractions (Figure $7 \mathrm{a}, \mathrm{b}$ ). Both isolated $78^{f / f}$ and $c 78^{f / f}$ mammary epithelial cells were able to repopulate glands upon transplantation into de-epithelialized hosts in multiple attempts (Figure 7c). Furthermore, flow cytometric analysis of isolated cells from repopulated $c 78^{f / f}$ mammary glands demonstrated reconstitution of differentiated mammary lineages (Figure $7 \mathrm{~d}$ ). Genotyping of the MYO, MRU and luminal colony forming cells (CFC) enriched fractions from re-isolated $c 78^{f f}$ mammary transplant revealed retained Grp78 floxed alleles whose proportion was variable among the different cell types analyzed, with the highest rate of retention in the MRU and lowest in the MYO fraction (Figure 7e).

GRP78 is required for mammary gland regeneration. Since in vivo deletion of Grp78 in the mammary gland using MMTV-Cre in the transgenic setting was ineffective, we subsequently employed adenoviral-Cre as an alternative strategy to ablate Grp78 in isolated mouse epithelium cells followed by transplantation (Figure 8a). Briefly, isolated $78^{f / f}$ mammary epithelial stem/progenitor cells were transduced with viruses expressing GFP and Cre recombinase (Ad-GFP-Cre) or GFP alone (Ad-GFP-Con), cultured overnight and sorted for GFP prior to transplantation into de-epithelialized recipient mice (Figure $8 \mathrm{a}, \mathrm{b}$ ). Surface GRP78 positive cells were dramatically reduced in cells transduced with Ad-GFP-Cre, compared to Ad-GFP-Con, as confirmed by flow cytometry (Figure 8c). All Ad-GFP-Con transduced samples $(n=3)$ gave rise to repopulated mammary glands after 6 weeks, whereas none of the Ad-GFP-Cre samples $(\mathrm{n}=9)$ repopulated to mammary glands (Figure 8d). 
a

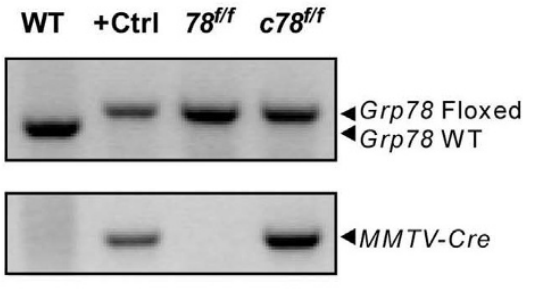

b

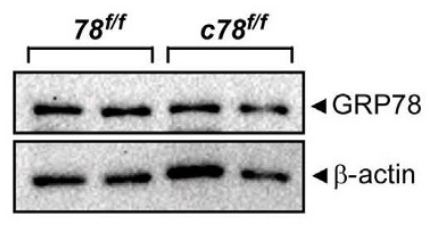

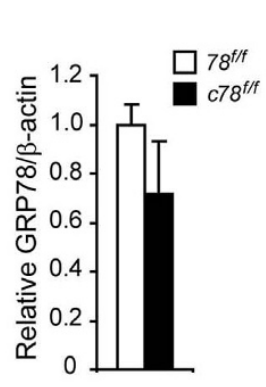

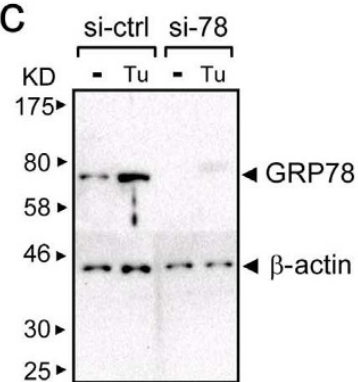

d

2 mos

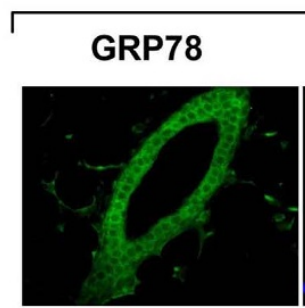

DAPI
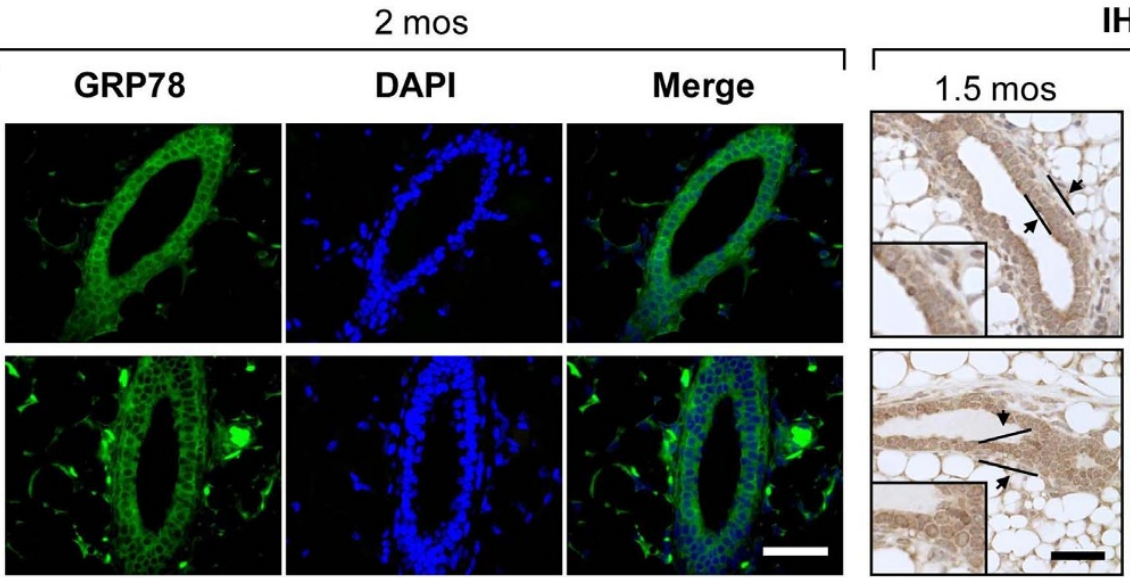

IHC
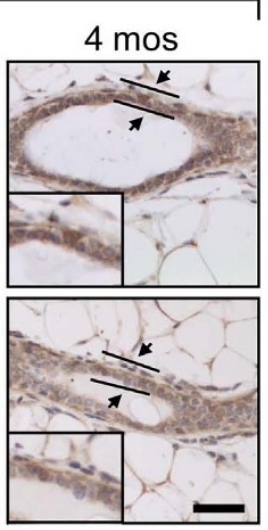

Figure $4 \mid$ Generation of MMTV-Cre, Grp $78^{f / f}$ mouse model. (a) Representative PCR genotyping results from tails of $78^{f f f}$ and $c 78^{f / f}$ mice. The alleles are indicated. Cropped gels were shown and the full-length gels were indicated in Supplementary Fig. 2b. (b) Representative Western blot detection of GRP78 levels in mammary epithelial cells isolated from $78^{f f}$ and $c 78^{f f f}$ glands at 2.5 months, with $\beta$-actin serving as loading control. Quantitation of GRP78 level after normalization to the $\beta$-actin level is shown on right $(n=3$ per genotype). Data are presented as mean \pm S.E. Cropped blots were shown and the full-length blots were indicated in Supplementary Fig. 3b. (c) Western blot analysis of lysates from A549 cells transfected with either si-control (si-ctrl) or si-Grp78 (si-78), followed by DMSO (-) or tunicamycin (Tu) treatment for 16 hours. (d) IF staining of GRP78 on 2 month old mammary glands from $78^{f f}$ and $c 78^{f f f}$ mice. The green and blue colors depict GRP78 and DAPI staining, respectively. IHC staining of GRP78 on 1.5 and 4 month old mammary glands from $78^{f f f}$ and $c 78^{f f f}$ mice. Brown color depicts GRP78 staining. Scale bars show $50 \mu \mathrm{m}$ and are applicable to all sections. Negative controls for staining were shown in Supplementary Fig. 4c, d.

\section{Discussion}

While the functions of GRP94 and GRP78 have been examined in various cell lines and tissues ${ }^{11}$, little is known about the requirement of these GRPs in mammary gland development and function. In this report, we created two novel mouse models bearing the MMTV-Cre recombinase transgene, coupled with either the Grp94/f or the $G r p 78^{f / f}$ alleles and examined the phenotypes of the compound mice. Additionally, we tested whether knockdown of GRP78 in isolated mammary epithelial stem/progenitor cells affected mammary gland regeneration. Our studies reveal several unexpected findings such that GRP94 may be dispensable for mammary gland development and that efficient genetic depletion of GRP78 in the mammary epithelial cells may not be achievable using the MMTV-Cre recombinase system in the transgenic setting. Nonetheless, GRP78 surface expression can be ablated in mammary epithelial cells following adenoviral-Cre transduction in vitro, which provides an alternative strategy to establish its role in mammary gland development in transplantation assays.

With regard to GRP94, as expected from the reported activity of the MMTV-Cre recombinase system in mammary epithelial cells starting from postnatal day 22 and becoming fully active by week $5^{32}$, GRP94 expression was substantially decreased in these cells in both 3 and 10 month old $c 94^{f / f}$ mice. However, despite the loss of GRP94 expression, no developmental defects in terms of ductal length and branching patterns were detected up to 11 months, nor did we observe any defect in TEB formation. Additionally, c $94^{f / f}$ glands showed normal alveolar development during lactation. The implication that GRP94 is not required for the developmental regulation of the mammary gland is intriguing since GRP94 is essential for IGF- 1 secretion and $\alpha 2$ integrin expression on the cell surface, both of which are known to influence mammary development. IGF-1 null female mice showed significant mammary development defect and IGF-1 mediated the action of GH in this process ${ }^{9}$. The $\alpha 2$ integrin subunit-deficient mice had impaired mammary branching morphogenesis ${ }^{6}$. Possible explanations include the ability of circulating IGF-1 produced by liver, muscle and mammary stroma to compensate for the loss of IGF-1 production in the $c 94^{f / f}$ mammary epithelium, and other integrins that may compensate for $\alpha 2$ integrin activity in vivo, as previously reported ${ }^{6}$. Furthermore, we observed that GRP94 deficiency in the mammary epithelium did not affect cellular proliferation.

In previous findings, acute elimination of GRP94 in the hematopoietic system caused an increase in hematopoietic stem cell (HSC) and granulocyte-monocyte progenitors in bone marrow, as well as the failure of the Grp94-knockout HSCs to express integrin $\alpha 4$ on the cell surface, associating with niche detachment ${ }^{34}$. Hyperproliferation of liver progenitor cells was also observed in liver-specific GRP94 knockout mouse model, correlating with disruption of cell-cell/cellmatrix interaction ${ }^{35}$. Knockout of GRP94 in the intestinal epithelium led to decrease in Wnt signaling, gut proliferation defect and postnatal death ${ }^{36}$. On the other hand, GRP94 is dispensable for the growth of embryonic stem cells ${ }^{33}$, the viability of Purkinje cells in the mouse cerebellum ${ }^{28}$ and B-cell development ${ }^{14}$. Tissue specific modes of action or requirements for GRP94 may have significant 
a
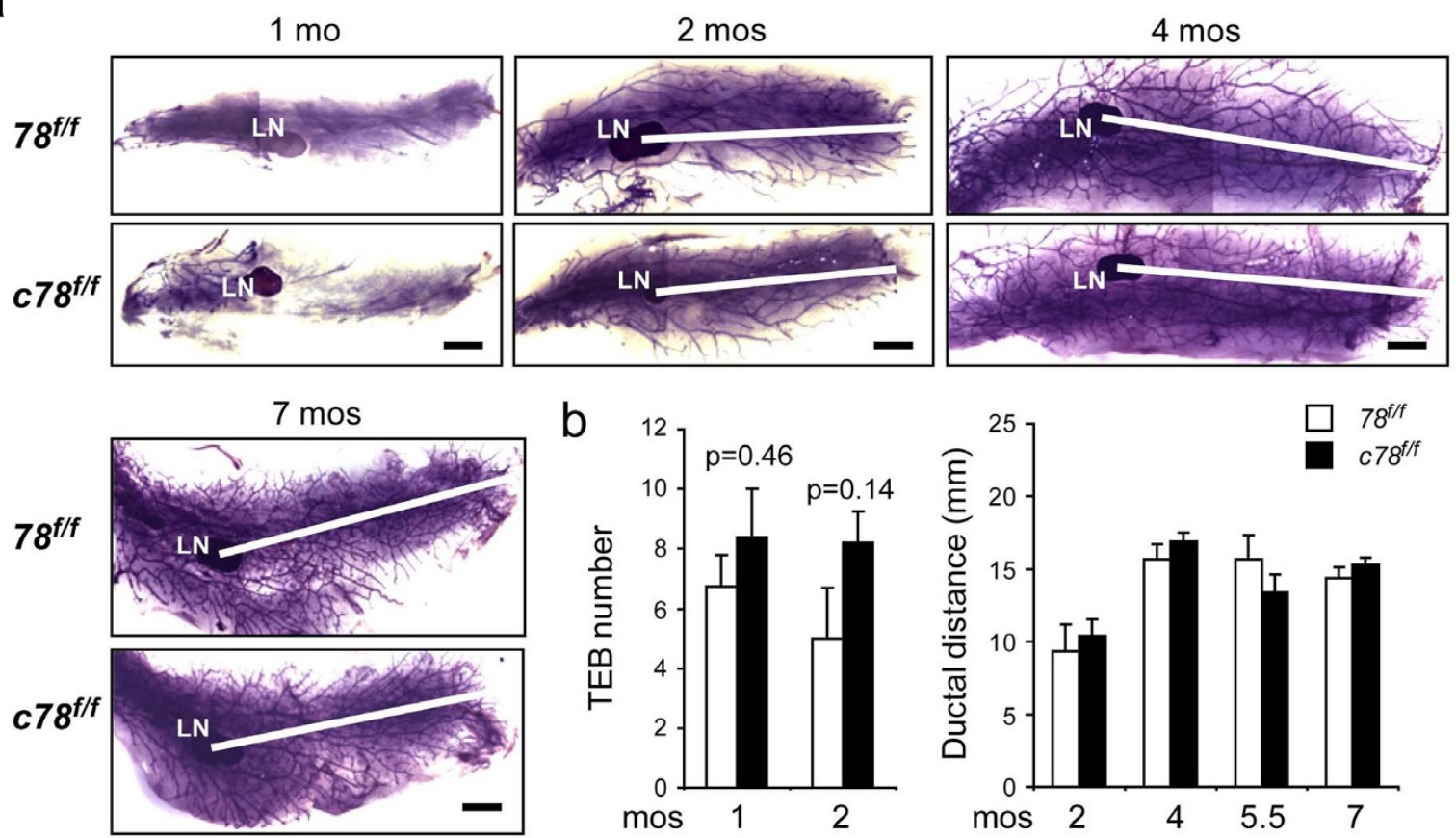

Figure $5 \mid$ Ductal morphogenesis of $c 78^{f / f}$ mammary glands. (a) Mammary glands from $78^{f f f}$ and $c 78^{f / f}$ mice at $1,2,4$ and 7 months were whole-mounted and representative mammary glands for each time point was presented. White bars denote the length between center of the lymph node and distal end of the longest duct in $78^{f / f}$ and $c 78^{f / f}$ glands. LN, lymph node. Scale bars show $2 \mathrm{~mm}$ and are applicable to all sections. (b) Quantitation of TEB number and ductal length of $78^{f f}$ and $c 78^{f f f}$ mammary glands at indicated times. For each genotype, $\mathrm{n}$ varies from 3 to 6 . Data are presented as mean \pm S.E.

a

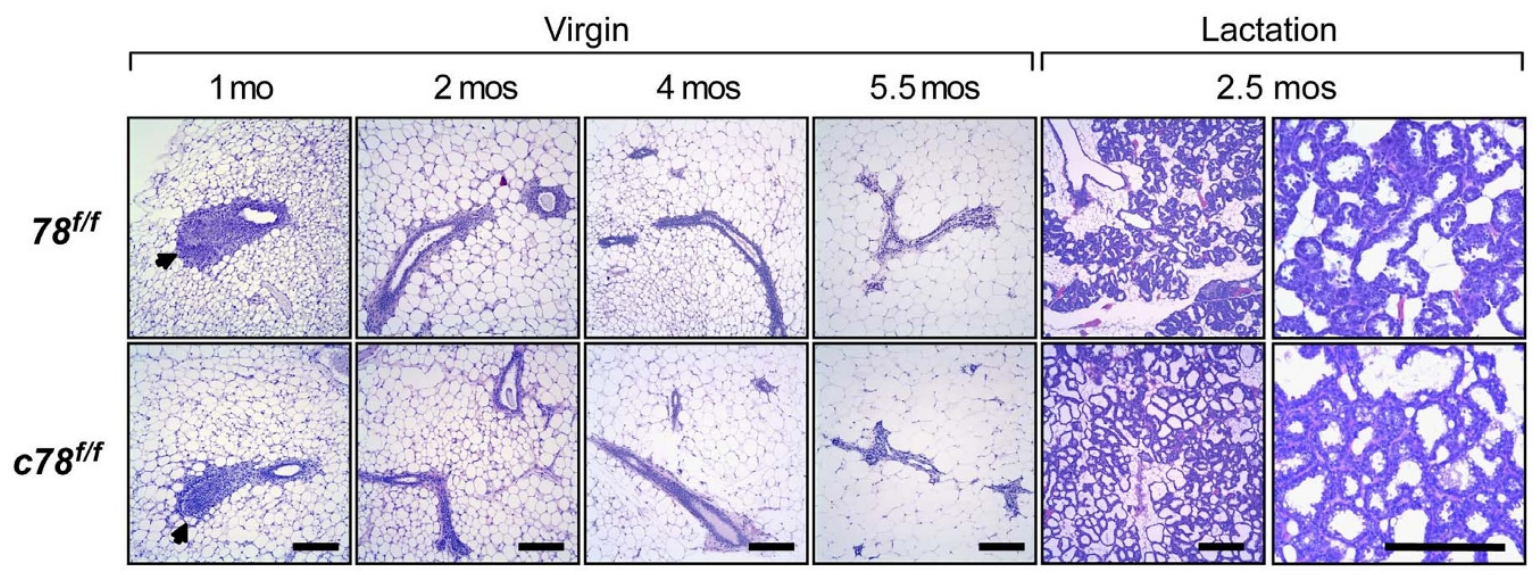

b
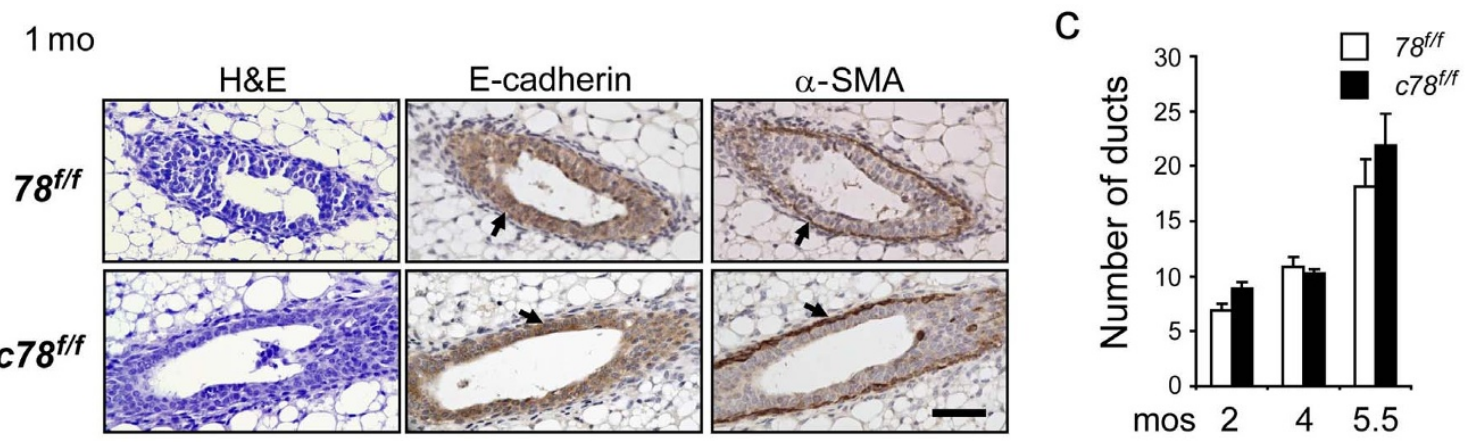

Figure 6 Morphology of $c 78^{f / f}$ mammary epithelium. (a) H\&E staining of $78^{f f f}$ and $c 78^{f f f}$ mammary glands from $1,2,4$ and 5.5 month old virgin mice and 2.5 month old lactating mice on day 7 after parturition. Arrows denote TEBs. Scale bars show $200 \mu \mathrm{m}$ and are applicable to all sections. (b) IHC staining of E-cadherin and $\alpha$-smooth muscle actin $\left(\alpha\right.$-SMA) on serial sections from mammary glands of 1 month old $78^{f / f}$ and $c 78^{f f f}$ mice, with corresponding H\&E staining. Brown color depicts E-cadherin or $\alpha$-SMA staining. Arrows denote positive cells. Scale bars show $50 \mu \mathrm{m}$ and are applicable to all sections. Negative controls for staining were shown in Supplementary Fig. 4e, f. (c) Quantitation of numbers of ducts from $78^{f f f}$ and $c 78^{f f f}$ mammary glands at 2, 4 and 5.5 months. For each genotype, n varies from 3 to 4 . 
a

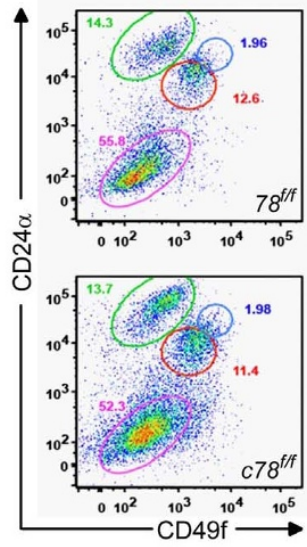

d

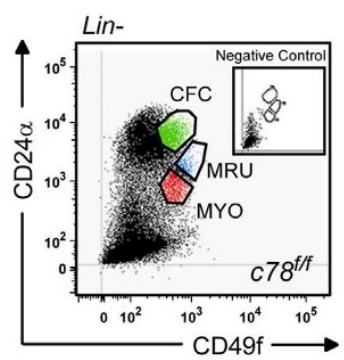

b

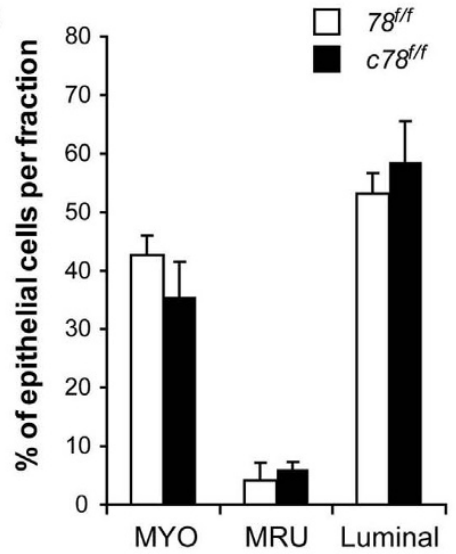

e

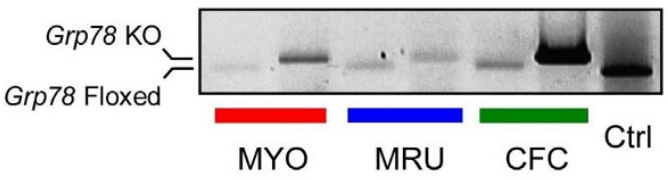

C
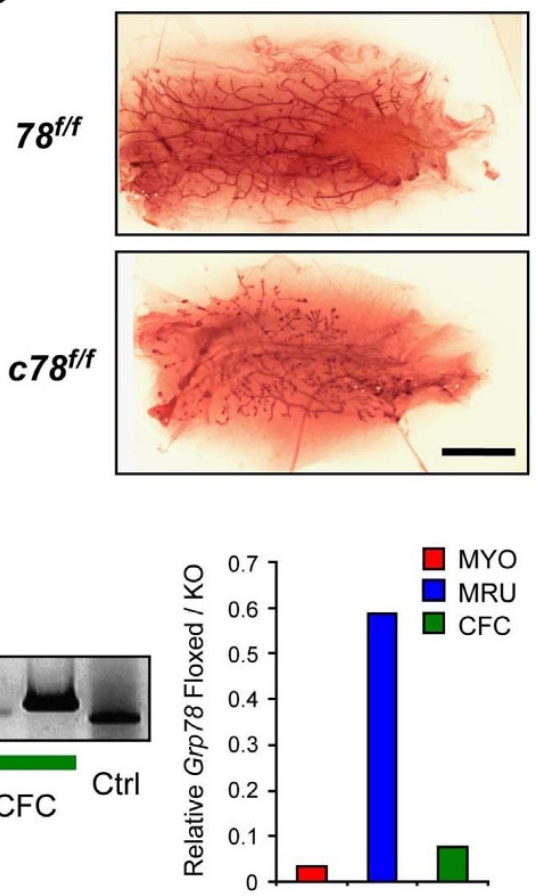

Figure $7 \mid$ Analysis of sub-lineages of $c 7 \boldsymbol{8}^{f / f}$ mammary glands and efficiency of conditional gene recombination. (a) Representative flow cytometric analyses of virgin mammary glands in $78^{f f f}$ (top panel) and $c 78^{f f f}$ mice (bottom panel) for surface markers (CD24 $\alpha$ and CD49f) that distinguish myoepithelial (MYO, red circle), mammary repopulating unit (MRU, blue circle) and luminal epithelial cells (green circle) enriched fractions from stroma cells (pink circle). (b) Percentage of MYO, MRU and luminal epithelial cells (luminal) fractions in $78^{f / f}$ and $c 78^{f / f}$ mammary epithelial cells ( $\mathrm{n}=3$ per genotype). (c) Representative pictures of mammary transplant outgrowths from epithelial cells isolated from $78^{f f f}$ and $c 78^{f f f}$ glands. There were outgrowths of both groups ( $\mathrm{n}=3$ per genotype). Scale bar shows $1 \mathrm{~cm}$ and is applicable to all sections. (d) Flow cytometric analysis of re-isolated epithelial cells from $c 78^{f / f}$ mammary transplant. Red dots: MYO; Blue dots: MRU; Green dots: luminal colony forming cells (CFC). (e) Genotyping of Grp78 knockout and floxed alleles in the MYO, MRU and CFC enriched fractions shown in panel d. The relative ratios of signal intensities for the nonrecombined (floxed) vs. recombined (KO) Grp78 allele were shown on right. Cropped gels were shown and the full-length gels were indicated in Supplementary Fig. 2c.

clinical implications. Expression of GRP94 in human breast carcinoma samples is significantly higher than that in normal breast tissue $^{37}$. Recently, it was reported that GRP94 is important in regulating plasma membrane HER2 signaling in HER2-overexpressed breast cancer cell lines ${ }^{38}$. This suggests that GRP94 could be a new target in treatment of patients with HER-2 overexpression and results of our genetic knockout model imply that targeting GRP94 in breast cancer treatment may not affect normal breast tissue.

Unexpectedly, GRP78 expression in the $c 78^{f / f}$ mammary epithelium was only slightly diminished compared to wild-type level. Correspondingly, no development or functional abnormalities were detected in virgin $c 78^{f / f}$ glands up to 7 months of age or lactating $c 78^{f / f}$ mammary glands. MYO, MRU and luminal epithelial cells enriched fractions isolated from $78^{f f f}$ and $c 78^{f / f}$ mammary glands showed no difference in distribution of these sub-lineages. Mammary transplants of epithelial cells isolated from $78^{f / f}$ and $c 78^{f / f}$ glands displayed similar take-rates, corresponding with incomplete and differential excision of the Grp78 floxed alleles in various lineages of epithelial cells re-isolated from $c 78^{f / f}$ mammary outgrowth. This raises the important question of whether the deletion of GRP78 was unsuccessful in the $c 78^{f / f}$ glands. While we cannot formally rule out the MMTV-Cre recombinase is specifically inefficient in deleting the $G r p 78^{f / f}$ alleles in the mammary epithelium, this is unlikely since the same Cre system was able to knockout the Grp94/f alleles in parallel experiments, and the same $G r p 78^{f / f}$ alleles had been deleted in the many different cell types and organs in previous studies ${ }^{26,29,39,40}$. An alternative explanation is that the intrinsic efficiency of MMTVCre may be similar in the Grp $78^{f / f}$ and Grp9 $94^{f / f}$ contexts but that selective pressure against GRP78 loss leads to repopulation of the epithelium by cells that retain the un-recombined alleles in $\mathrm{c} 78^{\mathrm{f} / f}$ mice. In support of this possibility, in an intestine-specific GRP78 knockout mouse model, recombination efficiency was high at day 1 post-induction of Cre recombinase, but there was increasing presence of non-recombined cells from day $3^{41}$. Repopulation of nonrecombined cells was also observed in an oesophagus knockout model of GRP78 ${ }^{42}$. Additionally, in a mouse model with conditional c-Myc knockdown in intestines, a similar pattern of repopulation was observed ${ }^{43}$. Although we cannot infer exact ratios between recombined and non-recombined alleles in our study due to potential differences in PCR efficiency using different primers, the changing ratios indicate a differing excision rate in the various mammary epithelial cell sub-populations. While it is not possible to conclude that mammary epithelial cells tolerate bi-allelic Grp78 deletion, these data suggest this is more likely in myoepithelial cells than mammary stem cells.

As an alternative strategy, we transduced isolated $78^{f / f}$ mammary epithelial stem/progenitor cells with Ad-GFP-Cre or Ad-GFP-Con and performed transplantation assays. Cells transduced with AdGFP-Cre failed to regenerate the mammary glands, associating with the loss of GRP78 surface expression. These data suggest that GRP78 plays an important role in stem cell function and homeostasis in the mammary gland. These findings are consistent with our recent discovery that cell surface GRP78 is a functional marker of fetal and adult mammary stem cells that mediates their responsiveness to the stem cell-associated factor Cripto ${ }^{44}$. In summary, we demonstrated here that although both GRP78 and GRP94 are key chaperones in the 
a

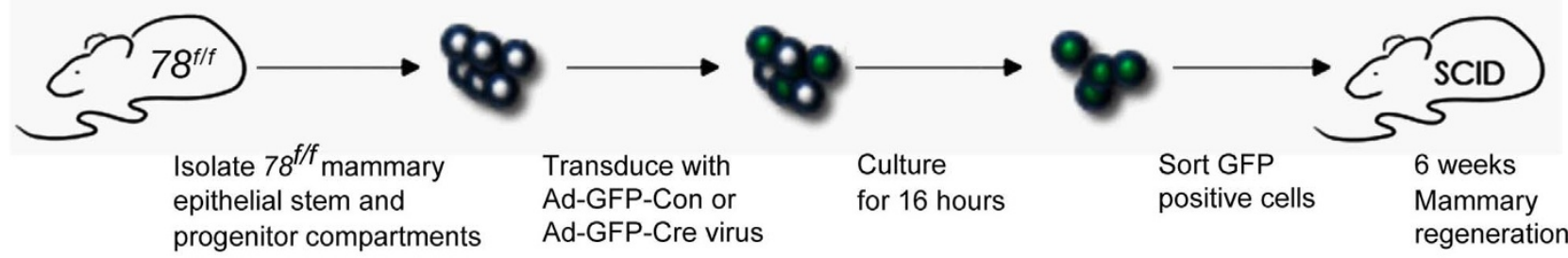

b

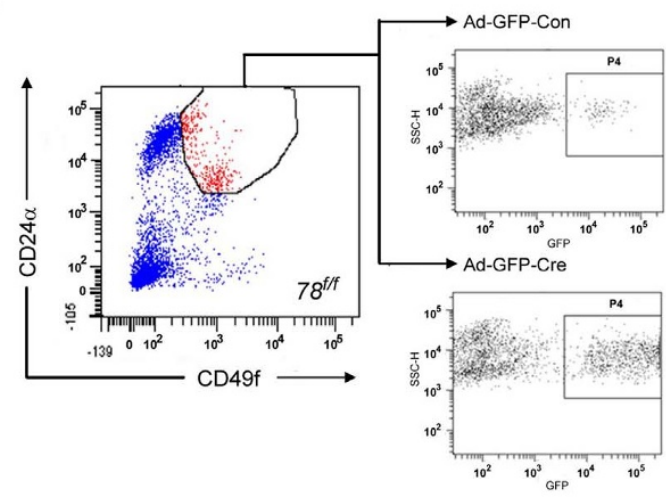

C

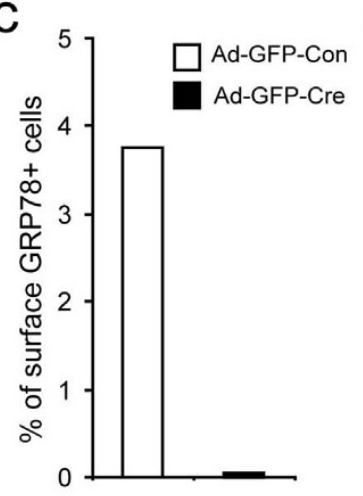

d

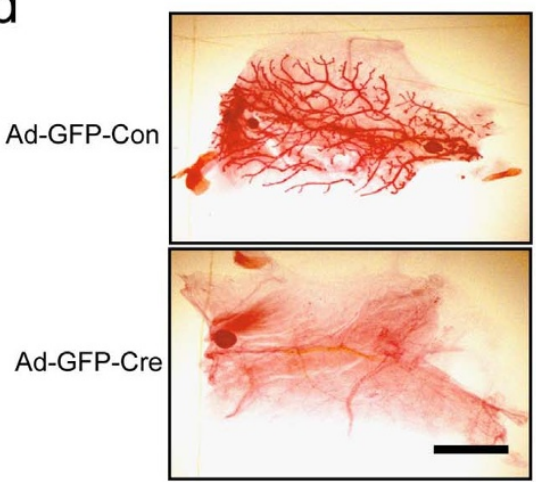

Figure $8 \mid$ GRP78 is required for mammary gland regeneration. (a) Schematic of adenovirus-Cre mediated recombination of Grp78 floxed alleles and mammary transplantation. (b) Sorted adult mammary epithelial stem and progenitor cells population (red) isolated by FACS from $78^{f / f}$ adult glands were transduced ex vivo with adenoviral vectors carrying GFP (Ad-GFP-Con) or GFP and Cre recombinase (Ad-GFP-Cre). GFP positive cells were sorted 16 hours later and used to inoculate de-epithelialized recipient glands. (c) Quantitation of flow cytometric analysis of surface GRP78 positive cells from transplanted Ad-GFP-Con and Ad-GFP-Cre mammary epithelial cells following 12 weeks outgrowth. (d) Representative whole mount mammary glands 6 weeks after transplantation of $78^{f / f}$ mammary epithelial cells transduced with Ad-GFP-Con or Ad-GFP-Cre. Ad-GFP-Con: $\mathrm{n}=3$; Ad-GFP-Cre: $\mathrm{n}=9$. Scale bar shows $1 \mathrm{~cm}$ and is applicable to all sections.

ER, they serve non-overlapping functions in the mouse mammary epithelial cells and that GRP78, but not GRP94, is critical for mammary gland development and regeneration.

\section{Methods}

Mouse models. All protocols for animal use and euthanasia were reviewed and approved by the University of Southern California Institutional Animal Care and Use Committee. All experiments were performed in accordance with the approved guidelines. MMTV-Cre mouse ${ }^{31}$ (line D, Jackson Laboratories) was mated with $94^{f / f}$ mice ${ }^{33}$ to generate $c 94^{f /+}$ offspring. Breeding between $94^{f / f}$ and $c 94^{f /+}$ mice yielded $c 94^{f / f}$ mice used in the experiments. $c 94^{f / f}$ and littermate control $94^{f / f}$ mice were expanded through the mating between $94^{f / f}$ and $c 94^{f / f}$ mice. The generation and characterization of $78^{f f f}$ mice were described previously ${ }^{25} . c 94^{f / f}$ mice were bred with $78^{f / f}$ mice to generate $c 94^{f /+} 78^{f /+}$ mice. Breeding between $c 94^{f /+} 78^{f /+}$ and $78^{f / f}$ mice yielded $c 78^{f / f}$ mice used in this study. The $c 78^{f / f}$ and littermate control $78^{f / f}$ mice were expanded through the mating between $c 78^{f / f}$ and $78^{f / f}$ mice. Genotyping was carried out as previously described for various Grp94, Grp78 and Cre alleles ${ }^{25,33}$. Virgin mice were used except where indicated. To synchronize the estrous cycle, pregnant mare's serum gonadotropin (PMSG) was injected 48 hours before euthanasia of mice to synchronize estrous cycle, if indicated in figure legends. For lactation, female mice were mated with male mice and mammary glands were collected 7 days after the pups were born.

H\&E, immunofluorescent and immunohistochemical staining. Mouse mammary glands were isolated and fixed overnight in $10 \%$ buffered formalin followed by embedding in paraffin. Paraffin-embedded tissues were sectioned and stained with hematoxylin and eosin (H\&E). The immunofluorescent and immunohistochemical staining were performed as described previously ${ }^{29}$. Paraffin sections were incubated at $4{ }^{\circ} \mathrm{C}$ overnight with primary antibodies against GRP94 (Enzo Life Sciences, 1:200), GRP78 (H-129, Santa Cruz, $1: 100)$, PCNA (BD BioScience, $1: 100)$, E-cadherin (BD BioScience, $1: 150$ ) or $\alpha$-SMA (Sigma, $1: 2000$ ). For immunofluorescent staining, the slides were mounted with VECTASHIELD mounting medium with DAPI (Vector Laboratories).

Tissue extracts, cell transfection and immunoblotting. Mammary epithelial cells were isolated for whole cell extracts as previously described ${ }^{45}$. Mammary glands (\#3 and \#4) were collected, minced and digested at $37^{\circ} \mathrm{C}$ for 2 hours in DMEM/F-12 media containing collagenase (Roche Applied Science, $2 \mathrm{mg} / \mathrm{ml}$ ) and hyaluronidase (Sigma, 100 units/ml), followed by washes with DMEM/F-12 media.
HBL100-HER2 cells were maintained in DMEM media and A549 cells were maintained in F-12K media, and both media were supplemented with $10 \%$ FBS and $1 \%$ penicillin/streptomycin. For knockdown of GRP94, the siRNA against Grp94 (si94) is $5^{\prime}$-aucugggacaagcgaguuuuu- $3^{\prime}$; for knockdown of GRP78, the siRNA against Grp78 (si-78) is 5' -ggagcgcauugauacuagatt- $3^{\prime}$; the control siRNA (si-ctrl) is $5^{\prime}$-aaggagacguauagcaacggu- $3^{\prime}$. HBL100-HER2 cells were transfected with si-ctrl or si-94 using Lipofectamine 2000 transfection reagent (Invitrogen) for 48 hours, according to the manufacturer's instructions. A549 cells were transfected with si-ctrl or si-78 using Lipofectamine RNAiMAX transfection reagent (Invitrogen), following the manufacturer's instructions. Seventy-two hours after transfection of siRNA, A549 cells were treated with $1.5 \mu \mathrm{g} / \mathrm{ml}$ tunicamycin or DMSO for 16 hours before harvesting.

Tissues or cells were homogenized in ice-cold RIPA buffer containing cocktails of proteinase inhibitors and phosphatase inhibitors (Pierce) and Western blotting were performed by standard protocol ${ }^{40}$. Primary antibodies used were rat monoclonal antiGRP94 (Enzo Life Sciences, 1:2000), rabbit polyclonal anti-GRP78 (H-129, Santa Cruz, $1: 1000$ ), mouse monoclonal anti-GPR78 (BD BioScience, $1: 1000$ ) and mouse monoclonal anti- $\beta$-actin (Sigma, $1: 5000)$. Rabbit polyclonal anti-GRP78 (H-129) antibody was used in the Western blotting for validating the GRP78 antibody for immunostaining.

Whole-mount staining, ductal distance and number measurement. Inguinal mouse mammary glands were extracted from female virgin mice at different ages. The detailed protocol about whole-mount hematoxylin staining of mammary glands was described previously ${ }^{46}$. In brief, glands were fixed in $10 \%$ neutral buffered formalin for 24 hours, followed by 48 hours acetone treatment. Tissues were then washed in $100 \%$ and $95 \%$ ethanol for 1 hour before the overnight staining with the hematoxylin staining solution. After that, tissues were washed with distilled $\mathrm{H}_{2} \mathrm{O}, 70 \%, 95 \%, 100 \%$ ethanol (three times) and xylene (three times) for 1 hour sequentially. Alternatively, glands were stained according to a similar procedure using carmine aluminum sulfate (Sigma). Finally, the stained tissues were placed in glass vials with methyl salicylate for permanent storage. For ductal distance measurement, the ductal length was determined by measuring the distance between the center of the lymph node and the end of the longest duct ${ }^{47}$. At various time intervals, the number of ducts in the mammary glands was measured from three random fields per section at $40 \times$ magnification for each genotype.

Mammary cell preparation, flow cytometric analysis and mammary transplant. Adult mammary glands were obtained from 3-month old nulliparous $78^{f / f}$ and $c 78^{f / f}$ mice. Minced adult mammary glands were digested for 14 hours at $37^{\circ} \mathrm{C}$ in EpiCult-B media containing B supplement, collagenase and hyaluronidase. All reagents were 
purchased from Stem Cell Technologies (SCT) unless otherwise specified. Mammary organoids resulting from overnight digestion were processed as previously

described ${ }^{48}$. Adult single cells were incubated with the following antibodies: Fc receptor antibody, biotinylated CD31, biotinylated CD45, biotinylated TER119, streptavidin-PerCP-Cy5.5 (BD Biosciences), CD24-PE (M1/69) and CD49f-FITC (GoH3). Cell sorting was carried out on FACS ARIA II cell sorter (Becton Dickinson). The surgical technique to clear the mammary fat pads and to conduct mammary transplantation has been well established ${ }^{49}$. Briefly, the inguinal mammary glands of 21 day-old CB17-SCID recipients were surgically removed and the cleared (epithelialfree) mammary fat pads left behind served as the transplantation sites. Transplants were carried out using 1,000 cells in the inoculum. Transplanted glands were removed from the recipients 12 weeks post-surgery for mammary repopulation evaluation unless otherwise noted for mammary repopulation evaluation. The excised glands were laid onto slides as whole-mounts or processed as above for FACS. Ductal structures extending through $\geq 50 \%$ of the fat pad in whole-mounts or flow cytometric profiles containing both luminal and myoepithelial populations were scored as positive for mammary repopulation.

Where viral transduction is indicated, DAPI-Lin-CD24 $\alpha+$ CD $49 f+$ mammary epithelial cells isolated from $78^{f / f}$ mice were sorted and transduced by $60 \mathrm{~min}$ spinoculation with adenoviral vectors expressing Ad-GFP-Cre or Ad-GFP-Con (MOI = 5). After overnight culture in DMEM/F12 media supplemented with 5\% horse serum, $10 \mu \mathrm{g} / \mathrm{ml}$ insulin, $20 \mathrm{ng} / \mathrm{ml} \mathrm{EGF}, 100 \mathrm{ng} / \mathrm{ml}$ cholera toxin, $0.5 \mu \mathrm{g} / \mathrm{ml}$ hydrocortisone and $10 \mu \mathrm{g} / \mathrm{ml}$ Ciprofloxacin, the cells were sorted for GFP expression and transplanted into de-epithelialized recipients as above. The excised glands were processed for whole-mounts or flow cytometric analyses as described above in combination with rabbit anti-GRP78 antisera produced previously ${ }^{44}$ and APC conjugated antirabbit secondary antibodies.

Statistical analyses. A 2-tailed Student's t-test was applied for all pairwise comparisons. Data are expressed as mean \pm S.E.

1. Macias, H. \& Hinck, L. Mammary gland development. Wiley Interdiscip Rev Dev Biol 1, 533-557 (2012)

2. Hennighausen, L. \& Robinson, G. W. Information networks in the mammary gland. Nat. Rev. Mol. Cell Biol. 6, 715-725 (2005).

3. Stein, T., Salomonis, N. \& Gusterson, B. A. Mammary gland involution as a multistep process. J Mammary Gland Biol Neoplasia 12, 25-35 (2007).

4. Muschler, J. \& Streuli, C. H. Cell-matrix interactions in mammary gland development and breast cancer. Cold Spring Harb Perspect Biol 2, a003202 (2010).

5. Lambert, A. W., Ozturk, S. \& Thiagalingam, S. Integrin signaling in mammary epithelial cells and breast cancer. ISRN Oncol 2012, 493283 (2012).

6. Chen, J., Diacovo, T. G., Grenache, D. G., Santoro, S. A. \& Zutter, M. M. The alpha(2) integrin subunit-deficient mouse: a multifaceted phenotype including defects of branching morphogenesis and hemostasis. Am J Pathol 161, 337-344 (2002).

7. Gallego, M. I. et al. Prolactin, growth hormone, and epidermal growth factor activate Stat 5 in different compartments of mammary tissue and exert different and overlapping developmental effects. Dev Biol 229, 163-175 (2001).

8. Silberstein, G. B., Van Horn, K., Shyamala, G. \& Daniel, C. W. Essential role of endogenous estrogen in directly stimulating mammary growth demonstrated by implants containing pure antiestrogens. Endocrinology 134, 84-90 (1994).

9. Ruan, W. \& Kleinberg, D. L. Insulin-like growth factor I is essential for terminal end bud formation and ductal morphogenesis during mammary development. Endocrinology 140, 5075-5081 (1999).

10. Schroder, M. \& Kaufman, R. J. The mammalian unfolded protein response. Annu Rev Biochem 74, 739-789 (2005).

11. Ni, M. \& Lee, A. S. ER chaperones in mammalian development and human diseases. FEBS Lett. 581, 3641-3651 (2007).

12. Lee, A. S. Glucose-regulated proteins in cancer: molecular mechanisms and therapeutic potential. Nat. Rev. Cancer 14, 263-276 (2014).

13. Eletto, D., Dersh, D. \& Argon, Y. GRP94 in ER quality control and stress responses. Semin. Cell Dev. Biol. 21, 479-485 (2010).

14. Liu, B. \& Li, Z. Endoplasmic reticulum HSP90b1 (gp96, grp94) optimizes B-cell function via chaperoning integrin and TLR but not immunoglobulin. Blood 112, 1223-1230 (2008)

15. Yang, Y. \& Li, Z. Roles of heat shock protein gp96 in the ER quality control: redundant or unique function? Mol. Cells 20, 173-182 (2005).

16. Staron, M. et al. gp96, an endoplasmic reticulum master chaperone for integrins and Toll-like receptors, selectively regulates early $\mathrm{T}$ and $\mathrm{B}$ lymphopoiesis. Blood 115, 2380-2390 (2010)

17. Barton, E. R. et al. Deletion of muscle GRP94 impairs both muscle and body growth by inhibiting local IGF production. FASEB J. 26, 3691-3702 (2012).

18. Ma, Y. \& Hendershot, L. M. The role of the unfolded protein response in tumour development: friend or foe? Nat. Rev. Cancer 4, 966-977 (2004).

19. Wang, M., Wey, S., Zhang, Y., Ye, R. \& Lee, A. S. Role of the unfolded protein response regulator GRP78/BiP in development, cancer, and neurological disorders. Antioxid. Redox Signal. 11, 2307-2316 (2009).

20. Ni, M., Zhang, Y. \& Lee, A. S. Beyond the endoplasmic reticulum: atypical GRP78 in cell viability, signalling and therapeutic targeting. Biochem. J. 434, 181-188 (2011)
21. Shani, G. et al. GRP78 and Cripto form a complex at the cell surface and collaborate to inhibit transforming growth factor beta signaling and enhance cell growth. Mol. Cell. Biol. 28, 666-677 (2008).

22. Kelber, J. A. et al. Blockade of Cripto binding to cell surface GRP78 inhibits oncogenic Cripto signaling via MAPK/PI3K and Smad2/3 pathways. Oncogene 28, 2324-2336 (2009).

23. Gonzalez-Gronow, M., Selim, M. A., Papalas, J. \& Pizzo, S. V. GRP78: a multifunctional receptor on the cell surface. Antioxid. Redox Signal. 11, 2299-2306 (2009).

24. Zhang, Y. et al. Cancer cells resistant to therapy promote cell surface relocalization of GRP78 which complexes with PI3K and enhances $\mathrm{PI}(3,4,5) \mathrm{P} 3$ production. PLoS One 8, e80071 (2013).

25. Luo, S., Mao, C., Lee, B. \& Lee, A. S. GRP78/BiP is required for cell proliferation and protecting the inner cell mass from apoptosis during early mouse embryonic development. Mol. Cell. Biol. 26, 5688-5697 (2006).

26. Zhu, G. et al. GRP78 plays an essential role in adipogenesis and postnatal growth in mice. FASEB J. 27, 955-964 (2013)

27. Wey, S., Luo, B. \& Lee, A. S. Acute inducible ablation of GRP78 reveals its role in hematopoietic stem cell survival, lymphogenesis and regulation of stress signaling. PLoS One 7, e39047 (2012).

28. Wang, M. et al. Essential role of the unfolded protein response regulator GRP78 $\mathrm{BiP}$ in protection from neuronal apoptosis. Cell Death Differ. 17, 488-498 (2010)

29. Fu, Y. et al. Pten null prostate tumorigenesis and AKT activation are blocked by targeted knockout of ER chaperone GRP78/BiP in prostate epithelium. Proc. Natl. Acad. Sci. U.S.A. 105, 19444-19449 (2008).

30. Pfaffenbach, K. T. et al. GRP78/BiP is a novel downstream target of IGF-1 receptor mediated signaling. J. Cell. Physiol. 227, 3803-3811 (2012).

31. Wagner, K. U. et al. Cre-mediated gene deletion in the mammary gland. Nucleic Acids Res. 25, 4323-4330 (1997).

32. Wagner, K. U. et al. Spatial and temporal expression of the Cre gene under the control of the MMTV-LTR in different lines of transgenic mice. Transgenic Res. 10, 545-553 (2001)

33. Mao, C. et al. Targeted mutation of the mouse Grp94 gene disrupts development and perturbs endoplasmic reticulum stress signaling. PLoS One 5, e10852 (2010).

34. Luo, B. et al. The endoplasmic reticulum chaperone protein GRP94 is required for maintaining hematopoietic stem cell interactions with the adult bone marrow niche. PLoS One 6, e20364 (2011).

35. Chen, W. T. et al. Liver-specific knockout of GRP94 in mice disrupts cell adhesion, activates liver progenitor cells, and accelerates liver tumorigenesis. Hepatology 59, 947-957 (2014)

36. Liu, B. et al. Essential roles of grp94 in gut homeostasis via chaperoning canonical Wnt pathway. Proc. Natl. Acad. Sci. U.S.A. 110, 6877-6882 (2013).

37. Hodorova, I. et al. Gp96 and its different expression in breast carcinomas. Neoplasma 55, 31-35 (2008).

38. Patel, P. D. et al. Paralog-selective Hsp90 inhibitors define tumor-specific regulation of HER2. Nat Chem Biol 9, 677-684 (2013).

39. Wey, S. et al. Inducible knockout of GRP78/BiP in the hematopoietic system suppresses Pten-null leukemogenesis and AKT oncogenic signaling. Blood 119 817-825 (2012)

40. Chen, W. T. et al. GRP78 as a regulator of liver steatosis and cancer progression mediated by loss of the tumor suppressor PTEN. Oncogene [Epub ahead of print] (2014)

41. Heijmans, J. et al. ER stress causes rapid loss of intestinal epithelial stemness through activation of the unfolded protein response. Cell. Rep. 3, 1128-1139 (2013).

42. Rosekrans, S. L. et al. ER stress induces epithelial differentiation in the mouse oesophagus. Gut [Epub ahead of print] (2014).

43. Muncan, V. et al. Rapid loss of intestinal crypts upon conditional deletion of the Wnt/Tcf-4 target gene c-Myc. Mol. Cell. Biol. 26, 8418-8426 (2006).

44. Spike, B. T. et al. CRIPTO/GRP78 signaling maintains fetal and adult mammary stem cells ex vivo. Stem Cell Reports 2, 427-439 (2014)

45. Grimm, S. L., Contreras, A., Barcellos-Hoff, M. H. \& Rosen, J. M. Cell cycle defects contribute to a block in hormone-induced mammary gland proliferation in CCAAT/enhancer-binding protein (C/EBPbeta)-null mice. J. Biol. Chem. 280, 36301-36309 (2005).

46. Brantley, D. M. et al. Nuclear factor-kappaB (NF-kappaB) regulates proliferation and branching in mouse mammary epithelium. Mol. Biol. Cell 12, 1445-1455 (2001).

47. Fiore, F. et al. Abnormal mammary gland development in MMTV-CBLC transgenic mouse. In Vivo 23, 225-228 (2009).

48. Spike, B. T. et al. A mammary stem cell population identified and characterized in late embryogenesis reveals similarities to human breast cancer. Cell Stem Cell 10, 183-197 (2012)

49. Deome, K. B., Faulkin, L. J., Jr., Bern, H. A. \& Blair, P. B. Development of mammary tumors from hyperplastic alveolar nodules transplanted into glandfree mammary fat pads of female C3H mice. Cancer Res. 19, 515-520 (1959).

\section{Acknowledgments}

We thank Drs. Louis Dubeau and Risheng Ye for helpful discussions and Daisy Flores for the gift of cell lysates. This work was supported in part by funding from the National 
Institutes of Health (R01 CA027607 and P01 AG034906) to ASL, and by the Clayton Medical Research Foundation, Inc. and DoD Award Number W81XWH-10-1-0891 to PCG, and by DoD Award Number W81XWH-10-1-0893 and The Breast Cancer Research Foundation to BTS. This work was also supported in part by Cancer Center Core Grant P30 CA014089-39 (ASL), P30 CA014195-38 (PCG) and P30 CA014195-40 (BTS). We thank the Tissue Imaging Core Facility of the USC Research Center for Liver Diseases (P30 DK048522) for microscopy. The content is solely the responsibility of the authors and does not necessarily represent the official views of the National Cancer Institute or the National Institutes of Health

\section{Author contributions}

A.L., G.Z. and M.W. conceived and designed the experiments; G.Z., B.S., M.W., J.S. and S.L. performed the experiments; G.Z., B.S. and M.W. analyzed the data; P.G. and S.C. contributed reagents/materials/analysis tools; G.Z., A.L. and J.S. wrote the paper.

\section{Additional information}

Supplementary information accompanies this paper at http://www.nature.com/ scientificreports

Competing financial interests: The authors declare no competing financial interests. How to cite this article: Zhu, G.Y. et al. Differential requirement of GRP94 and GRP78 in mammary gland development. Sci. Rep. 4, 5390; DOI:10.1038/srep05390 (2014).

(c) (i) (5) This work is licensed under a Creative Commons Attribution-NonCommercial-

cc) NoDerivs 4.0 International License. The images or other third party material in this article are included in the article's Creative Commons license, unless indicated otherwise in the credit line; if the material is not included under the Creative Commons license, users will need to obtain permission from the license holder in order to reproduce the material. To view a copy of this license, visit http:// creativecommons.org/licenses/by-nc-nd/4.0/ 